\title{
Unattended automated office blood pressure measurement - current evidence and the role in clinical practice
}

\author{
Marek Stopa ${ }^{1,2}$, Agnieszka Olszanecka', Marek Rajzer' \\ ${ }^{\prime} 1^{\text {st }}$ Department of Cardiology, Interventional Electrocardiology and Arterial Hypertension, Jagiellonian University Medical College, Kraków, Poland \\ ${ }^{2}$ Doctoral School of Medical and Health Sciences, Jagiellonian University Medical College, Kraków, Poland
}

\begin{abstract}
Arterial hypertension is a leading preventable cardiovascular risk factor. The definition and thresholds for the diagnosis of hypertension vary between European and American guidelines. That is mainly due to the widely known SPRINT trial in which unattended automated blood pressure measurements were used. This technique of blood pressure estimation requires a patient to be left alone in an office and then a programmed device measures blood pressure automatically. The absence of a health professional during the measurement helps to reduce or eliminate the "white coat" effect; therefore, values of blood pressure may be lower than in conventional office blood pressure measurements. There are premises that this technique can be a solid substitution for 24-hour ambulatory blood pressure measurements and that it can predict hypertension-mediated organ damage more accurately than standard techniques. However, due to the many methods in which measurement can be carried out, no universal protocol exists. More research is needed to evaluate the usefulness of unattended automated office blood pressure measurements in clinical practice.
\end{abstract}

Key words: arterial hypertension; blood pressure measurement; unattended automated blood pressure measurement; ambulatory blood pressure measurement; home blood pressure measurement, hypertension-mediated organ damage

Arterial Hypertens. 2021, vol. 25, no. 3, pages: 100-105

DOI: 10.5603/AH.a2021.0012

\section{Introduction}

Arterial hypertension is the leading preventable cardiovascular risk factor $[1,2]$. High blood pressure is responsible for $47 \%$ of cases of ischemic heart disease and $54 \%$ of stroke cases globally [3]. Hypertension also increases the risk of heart failure, atrial fibrillation, and chronic kidney disease [4]. Despite new advances and recommendations in the treatment of chronically elevated blood pressure (BP), such as the usage of single pill drug combinations
[5], many cases of hypertension are still suboptimally controlled. In Poland, for example, only $20.7 \%$ of hypertensive patients have their blood pressure in the recommended target range [6].

Another discussed issue is the definition of arterial hypertension. Threshold values of arterial hypertension are chosen arbitrarily because the association between blood pressure and cardiovascular risk is continuous [7]. According to the guidelines of the European Society of Hypertension and European Society of Cardiology, threshold values of arterial

\footnotetext{
Address for correspondence: Agnieszka Olszanecka, $1^{\text {st }}$ Department of Cardiology, Interventional Electrocardiology, and Hypertension, Jagiellonian University Medical College, 17 Kopernika Str, 30-688 Krakow, Poland, tel: + 481240021 11, fax: +48 1240021 17; e-mail: agnieszka.olszanecka@uj.edu.pl
}

This article is available in open access under Creative Common Attribution-Non-Commercial-No Derivatives 4.0 International (CC BY-NC-ND 4.0) license, allowing to download articles and share them with others as long as they credit the authors and the publisher, but without permission to change them in any way or use them commercially 
hypertension are a systolic blood pressure of 140 millimeters of Mercury ( $\mathrm{mm} \mathrm{Hg}$ ) and above, and a diastolic blood pressure of $90 \mathrm{mmHg}$ and above [5]. In contrast, the American College of Cardiology and American Heart Association defines arterial hypertension as a systolic blood pressure of 130 $\mathrm{mmHg}$ and above and a diastolic blood pressure of $80 \mathrm{mmHg}$ and above [8]. This discrepancy is caused primarily by the results of the SPRINT study, in which the method of unattended automated blood pressure measurements (UAOBPM) was used [9].

The aims of this article is to explore this method, discuss its advantages and disadvantages, its possible use in clinical practice, and its comparison to other, traditional methods such as conventional office blood pressure measurement or ambulatory blood pressure monitoring $(\mathrm{ABPM})$.

\section{The use of unattended automated office blood pressure measurement}

The main tenet of UAOBPM is to avoid or reduce the "white coat" effect - a phenomenon of the acute rise of blood pressure during measurement in the presence of medical professionals primarily due to anxiety [10]. This effect is common and it may be responsible for up to $50 \%$ of cases of white-coat hypertension, a condition in which $\mathrm{BP}$ values are above the threshold during the office BP measurements, while they are normal when measured at home or in ABPM [11]. Although white-coat hypertension is not a true arterial hypertension, patients with the former have a higher cardiovascular risk than normotensive patients [12]. This is why it is essential to distinguish between those 3 states. Home blood pressure measurements (HBPM) and ambulatory blood pressure monitoring are methods that can accomplish that [13]. It may be also useful to use unattended automated measurements. In the course of UAOBPM, a health professional places the blood pressure cuff and programs the device, then the patient is left alone in the office. After a specified amount of time, blood pressure will be measured automatically for a defined number of times [14]. On the market, there are many devices customized to unattended measurements that have been used in research. The list of available equipment is presented in Table 1.

The main concern of unattended measurements is that there are many variables and ways in which the device can be programmed. Programmable values are: a number of measurements, the time between measurements, and the delay before the first mea-
Table 1. Devices customized for unattended automated blood pressure measurements (UAOBPM)

\begin{tabular}{|l|}
\hline Omron HEM 907 [15] \\
Omron 907XL [14] \\
Omron HEM 9000Ai [16] \\
OMRON M10-IT [17] \\
Omron HEM-705CP [18] \\
Omron i-C10 [19] \\
Microlife WatchBP office [20] \\
BpTRU model BP300 [21] \\
Welch Allyn Connex Spot BP [22] \\
Mobil-O-Graph NG device [23] \\
CB-1805-B Biox [24] \\
Task Force Monitor, CNS Systems [25] \\
Dinamap ProCare DPC 400 Vital Signs Monitor [26]
\end{tabular}

surement. One must also decide if the values are displayed for the patients, or if the first measurement is discarded. Programmable variables and possible settings are presented in Table 2.

Therefore, there is currently not one universally-acceptable protocol for UAOBPM, although some studies have been performed to get the best settings. Studies of Myers and colleagues suggest that a 1- or 2-minute interval between measurements provides the best results $[27,28]$. For the number of measurements, Kronish et al. found that averaging two or three measurements gives the best precision of blood pressure estimation [29]. Similar conclusions were presented by Moore et al. [30]. In the office blood pressure measurements, Guidelines of the European Society of Hypertension state that patients should rest for 5 minutes before BP measurements commence [5]. This recommendation has been transferred to many protocols of UAOBPM, including the most famous SPRINT trial [9]. In fact, in the research of Colella et al. mean BP values were higher by $4 \mathrm{mmHg}$ for UAOBPM without a rest period in comparison to a standardized 5-minute delay before the first measurement [31].

Another difficulty of UAOBPM is the place in which the measurements are done. In some facilities, it may cause a problem as a patient needs to

Table 2. Possible setting of unattended automated blood pressure measurements (UAOBPM)

\begin{tabular}{|l|c|}
\hline Variable & Possible settings \\
\hline Number of measurements & $1,3,5,6$ \\
\hline Time between measurements & $0,5,1,2,5$ [minutes] \\
\hline Delay before the first measurement & $0,3,5,10$ [minutes] \\
\hline Results displayed for patients & Yes/No \\
\hline First measurements discarded & Yes/No \\
\hline
\end{tabular}


stay in an office for a few minutes alone, which may require an additional room. Research has been conducted to resolve this shortcoming. Armstrong et al. has shown that patients do not need to stay in the office for unattended measurements. Measurements taken in the waiting room without the presence of a medical professional were similar to these taken in the office while left alone [32]. Chambers et al. found that UAOBPM can be also performed at the community pharmacy with the same results as in the doctor's office [33]. It may resolve the important drawback of UAOBPM. Nevertheless, more research is needed to provide the best setting of programmable variables to obtain a universal UAOBPM protocol.

\section{Unattended automated office blood pressure measurement versus the classic office blood pressure measurement techniques}

In many research studies, it has been shown that unattended measurements are often associated with lower $\mathrm{BP}$ values than the readings conducted in the presence of a medical professional. Paini et al. found that attended measurements provide higher values than measurements taken without the presence of a health professional while using the same device, although this effect is not present for all patients [34].

A similar elimination of the white-coat effect was observed in the study of Myers et al. in which unattended systolic blood pressure was lower by 19 $\mathrm{mm} \mathrm{Hg}$ in comparison to measurements done by a hypertension specialist; however, both values were higher than those obtained by ABPM [35]. Beckett and Godwin achieved similar results; UAOBPM values were lower and more similar to ABPM than office measurements [36]. In the meta-analysis of Jegatheswaran et al., UAOBPM differed from ABPM only by $1.5 \mathrm{mmHg}$, suggesting that UAOBPM may be a solid ABPM substitution. UABOPM has been tested in routine clinical practice. In a multi-center study by Myers et al., UAOBPM values were comparable with ABPM and lower than those obtained during conventional measurements [37].

On the contrary, in the systematic review and meta-analysis of Kollias et al., differences between unattended and attended measurements were not statistically significant [38]. However, this paper included solely 10 studies that used a very strict, standardized protocol of both unattended and attended measurement. As authors concluded, lower values of the blood pressure during unattended measurements in comparison to conventional measurements seen in many other research studies are not dependent on whether a medical professional is present or absent, but it is due to minimization of common errors which are usually present during the office blood pressure measurements. In a systematic review and meta-analysis of Roerecke et al. which consisted of 31 articles, UAOBPM values were similar to ABPM recording, and as according to authors UAOBPM should be the preferred method of BP measurements in clinical practice [39]. Further research is needed to discover the true relationship between UAOBPM, ABPM, HBPM, and conventional office BP measurements.

\section{Prediction of hypertension mediated organ damage by UAOBPM}

Hypertension-mediated organ damage (HMOD) are specific changes caused by elevated blood pressure. Examples of HMOD are left ventricular hypertrophy, arterial wall thickening and albuminuria [40]. The early detection of HMOD can accelerate the initiation of pharmacotherapy in patients with grade 1 hypertension [5]. Few studies were performed to evaluate UAOBPM accuracy in the prediction of HMOD in comparison to standard methods of blood pressure measurement. In the study of Campbell et al., unattended measurements were better related to carotid intima-media thickness than auscultatory blood pressure measurements [41]. In contrast, Calompa et al. found no significant differences between correlations of blood pressure measured using either the attended or unattended method with respect to the intima-media thickness [42]. Salvetii et al. found that both attended and unattended blood pressure measurements were similarly correlated to left ventricular hypertrophy and intima-media thickness [43], while in the study of Andreadis et al., unattended measurements had a better correlation with left ventricular mass than attended measurements [20]. Additional research is needed to evaluate the usefulness of UAOBPM in the prediction of HMOD.

\section{Pros, cons and practical aspects of unattended automated office blood pressure measurements use}

UAOBPM method has its advantages and disadvantages. Some of them given by the various publications are listed in Table 3. 
Table 3. Advantages and Disadvantages of unattended automated blood pressure measurements (UAOBPM)

\begin{tabular}{|l|l|}
\hline Advantages & Disadvantages \\
\hline Reduction or elimination & Cost of the device \\
of the "white coat" effect & Increased time of measurement \\
Possible better accuracy in de- & Requirement of office space \\
tection of hypertension-mediated & No medical personnel present \\
organ damage & to detect errors in device \\
Better correlation between HBPM & measurements \\
and ABPM than attended measu- & No standardized protocol \\
rements & \\
Helps to avoid common errors & \\
during office blood pressure measu- \\
rements such as limited amount of \\
measurements
\end{tabular}

HBPM — home blood pressure measurements; ABPM — ambulatory blood pressure monitoring

UAOBPM is a rather new method and it is still not very well established in the application of hypertension management. The Canadian Society of Hypertension finds UAOBPM as a preferred method of measuring blood pressure. Thresholds for the diagnosis of hypertension using UAOBPM are 135 $\mathrm{mmHg}$ and above for systolic blood pressure, and 85 $\mathrm{mmHg}$ and above for diastolic blood pressure. The first measurement is taken by a health professional and the next measurements are automatic while the patient is left alone in the room [44].

In the guidelines of the European Society of Hypertension [5], and the Polish Society of Hypertension [45], there is only a small chapter dedicated to UAOBPM. It is stated that due to the small amount of research on this topic, threshold values are unclear and therefore, there are no specific recommendations of this method. The most recent Guidelines of the International Society of Hypertension as well as the Consensus of European Society of Hypertension consider UAOBPM as a more standardized assessment of blood pressure than standard office measurements, but thresholds for decisions are still uncertain. [46][47]

\section{Conclusions}

Unattended automated office blood pressure measurement is a new method of blood pressure assessment. It has its advantages and disadvantages in comparison to standard methods such as conventional office blood pressure measurements, home blood pressure measurements, or 24-hour ambulatory blood pressure measurements. Due to many variants of UAOBPM, further research is needed to provide a standard protocol for such measurements. More research is also needed to assess the accuracy of UAOBPM in the prediction of subclinical target organ damage caused by arterial hypertension. Nevertheless, UAOBPM has already been advised in the recent guidelines of many hypertension societies worldwide. Perhaps in the near future, with more available evidence, unattended measurements will be significantly highlighted in the guidelines of the Polish Society of Hypertension or the European Society of Hypertension and considered to be a preferred method of assessing blood pressure in patients.

\section{References}

1. O’Donnell MJ, Chin SL, Rangarajan S, et al. INTERSTROKE investigators. Global and regional effects of potentially modifiable risk factors associated with acute stroke in 32 countries (INTERSTROKE): a case-control study. Lancet. 2016; 388(10046): 761-775, doi: 10.1016/S0140-6736(16)30506-2, indexed in Pubmed: 27431356.

2. GBD 2017 Risk Factor Collaborators. Global, regional, and national comparative risk assessment of 84 behavioural, environmental and occupational, and metabolic risks or clusters of risks for 195 countries and territories, 1990-2017: a systematic analysis for the Global Burden of Disease Study. Lancet . 2018; 392(10159): 1923-1994, doi: 10.1016/S0140-6736(18)322256, indexed in Pubmed: 30496105 .

3. Lawes CMM, Vander Hoorn S, Rodgers A, et al. International Society of Hypertension. Global burden of bloodpressure-related disease, 2001. Lancet. 2008; 371(9623): 1513-1518, doi: 10.1016/S0140-6736(08)60655-8, indexed in Pubmed: 18456100.

4. Chobanian AV, Bakris GL, Black HR, et al. National Heart, Lung, and Blood Institute Joint National Committee on Prevention, Detection, Evaluation, and Treatment of High Blood Pressure, National High Blood Pressure Education Program Coordinating Committee. The Seventh Report of the Joint National Committee on Prevention, Detection, Evaluation, and Treatment of High Blood Pressure: the JNC 7 report. JAMA. 2003; 289(19): 2560-2572, doi: 10.1001/jama.289.19.2560, indexed in Pubmed: 12748199.

5. Williams B, Mancia G, Spiering W, et al. Authors/Task Force Members:, ESC Scientific Document Group . 2018 ESC/ESH Guidelines for the management of arterial hypertension. Eur Heart J. 2018; 39(33): 3021-3104, doi: 10.1093/eurheartj/ ehy339, indexed in Pubmed: 30165516.

6. Niklas A, Flotyńska A, Puch-Walczak A, et al. WOBASZ II investigators. Prevalence, awareness, treatment and control of hypertension in the adult Polish population - Multi-center National Population Health Examination Surveys - WOBASZ studies. Arch Med Sci. 2018; 14(5): 951-961, doi: 10.5114/ aoms.2017.72423, indexed in Pubmed: 30154875.

7. Ettehad D, Emdin CA, Kiran A, et al. Blood pressure lowering for prevention of cardiovascular disease and death: a systematic review and meta-analysis. Lancet. 2016; 387(10022): 957-967, doi: 10.1016/S0140-6736(15)01225-8, indexed in Pubmed: 26724178.

8. Whelton PK, Carey RM, Aronow WS, et al. 2017 ACC/AHA/ AAPA/ABC/ACPM/AGS/APhA/ASH/ASPC/NMA/PCNA Guideline for the Prevention, Detection, Evaluation, and Management of High Blood Pressure in Adults: Executive Summary: A Report of the American College of Cardiology/American Heart Association Task Force on Clinical Practice Guidelines. Hypertension. 2018; 71(6): 1269-1324, doi: 10.1161/ HYP.0000000000000066, indexed in Pubmed: 29133354.

9. Wright JT, Williamson JD, Whelton PK, et al. SPRINT Research Group. A Randomized Trial of Intensive versus Standard Blood- 
Pressure Control. N Engl J Med. 2015; 373(22): 2103-2116, doi: 10.1056/NEJMoa1511939, indexed in Pubmed: 26551272.

10. Ramli As, Halmey N, Teng Cl. White coat effect and white coat hypertension: one and the same? Malays Fam Physician. 2008; 3(3): 158-161, indexed in Pubmed: 25606143.

11. de la Sierra A, Vinyoles E, Banegas JR, et al. Prevalence and clinical characteristics of white-coat hypertension based on different definition criteria in untreated and treated patients. J Hypertens. 2017; 35(12): 2388-2394, doi: 10.1097/ HJH.0000000000001493, indexed in Pubmed: 28723880.

12. Huang Y, Huang W, Mai W, et al. White-coat hypertension is a risk factor for cardiovascular diseases and total mortality. J Hypertens. 2017; 35(4): 677-688, doi: 10.1097/HJH.0000000000001226, indexed in Pubmed: 28253216.

13. Mancia G, Bombelli M, Cuspidi C, et al. Cardiovascular Risk Associated With White-Coat Hypertension: Pro Side of the Argument. Hypertension. 2017; 70(4): 668-675, doi: 10.1161/HYPERTENSIONAHA.117.08903, indexed in Pubmed: 28847891.

14. Kjeldsen SE, Lund-Johansen P, Nilsson PM, et al. Unattended Blood Pressure Measurements in the Systolic Blood Pressure Intervention Trial: Implications for Entry and Achieved Blood Pressure Values Compared With Other Trials. Hypertension. 2016; 67(5): 808-812, doi: 10.1161/HYPERTENSIONAHA.116.07257, indexed in Pubmed: 27001295.

15. Asayama K, Ohkubo T, Rakugi H, et al. Japanese Society of Hypertension Working Group on the COmparison of Self-measured home, Automated unattended office and Conventional attended office blood pressure (COSAC) study. Comparison of blood pressure values-self-measured at home, measured at an unattended office, and measured at a conventional attended office. Hypertens Res. 2019; 42(11): 1726-1737, doi: 10.1038/s41440-019-02876, indexed in Pubmed: 31222188.

16. Salvetti M, Paini A, Bertacchini F, et al. Relationship between unattended and attended bp values and preclinical organ damage. J Hypertens. 2018; 36(Suppl 1): e112, doi: 10.1097/01. hjh.0000539295.55926.c5.

17. Polonia J, Baptista C, Silva J, et al. Unattended versus two attended, ambulatory and central blood pressure measurements in hypertensive patients with and without diabetes. Blood Press. 2019; 28(2): 99-106, doi: 10.1080/08037051.2019.1568184, indexed in Pubmed: 30669880.

18. Stergiou GS, Efstathiou SP, Argyraki CK, et al. Clinic, home and ambulatory pulse pressure: comparison and reproducibility. J Hypertens. 2002; 20(10): 1987-1993, doi: 10.1097/00004872200210000-00018, indexed in Pubmed: 12359977.

19. Al-Karkhi I, Al-Rubaiy R, Rosenqvist U, et al. Comparisons of automated blood pressures in a primary health care setting with selfmeasurements at the office and at home using the Omron i-C10 device. Blood Press Monit. 2015; 20(2): 98-103, doi: $10.1097 /$ MBP.0000000000000088, indexed in Pubmed: 25341027.

20. Andreadis EA, Geladari CV, Angelopoulos ET, et al. Attended and Unattended Automated Office Blood Pressure Measurements Have Better Agreement With Ambulatory Monitoring Than Conventional Office Readings. J Am Heart Assoc. 2018; 7(8), doi: 10.1161/JAHA.118.008994, indexed in Pubmed: 29627767.

21. Greiver M, White D, Kaplan DM, et al. Where should automated blood pressure measurements be taken? Pilot RCT of BpTRU measurements taken in private or nonprivate areas of a primary care office. Blood Press Monit. 2012; 17(3): 137-138, doi: 10.1097/MBP.0b013e328352ae44, indexed in Pubmed: 22561736.

22. Alpert BS. Clinical evaluation of the Welch Allyn SureBP algorithm for automated blood pressure measurement. Blood Press Monit. 2007; 12(4): 215-218, doi: 10.1097/ MBP.0b013e3280b0840d, indexed in Pubmed: 17625393.

23. Franssen PML, Imholz BPM. Evaluation of the Mobil-OGraph new generation ABPM device using the ESH criteria. Blood Press Monit. 2010; 15(4): 229-231, doi: $10.1097 /$ mbp.0b013e328339be38, indexed in Pubmed: 20658764.
24. Hong D, Su H, Li J, et al. The effect of physician presence on blood pressure. Blood Press Monit. 2012; 17(4): 145-148, doi: 10.1097/MBP.0b013e328355fe14, indexed in Pubmed: 22705861.

25. Martin CA, Cameron JD, Chen SS, et al. Two hour glucose post loading: a biomarker of cardiovascular risk in isolated clinic hypertension. J Hypertens. 2011; 29(4): 749-757, doi: 10.1097/ HJH.0b013e328342eeeb, indexed in Pubmed: 21192271.

26. Papademetriou V, Tsioufis C, Chung A, et al. Unobserved automated office BP is similar to other clinic BP measurements: A prospective randomized study. J Clin Hypertens (Greenwich). 2018; 20(10): 1411-1416, doi: 10.1111/jch.13371, indexed in Pubmed: 30272388.

27. Myers MG, Valdivieso M, Kiss A, et al. Comparison of two automated sphygmomanometers for use in the office setting. Blood Press Monit. 2009; 14(1): 45-47, doi: 10.1097/ MBP.0b013e32831e314f, indexed in Pubmed: 19190490.

28. Myers MG, Valdivieso M, Kiss A. Optimum frequency of office blood pressure measurement using an automated sphygmomanometer. Blood Press Monit. 2008; 13(6): 333-338, doi: 10.1097/ MBP.0b013e3283104247, indexed in Pubmed: 19020423.

29. Kronish IM, Edmondson D, Shimbo D, et al. A Comparison of the Diagnostic Accuracy of Common Office Blood Pressure Measurement Protocols. Am J Hypertens. 2018; 31(7): 827-834, doi: 10.1093/ajh/hpy053, indexed in Pubmed: 29897394.

30. Moore MN, Schultz MG, Nelson MR, et al. Identification of the Optimal Protocol for Automated Office Blood Pressure Measurement Among Patients With Treated Hypertension. Am J Hypertens. 2018; 31(3): 299-304, doi: 10.1093/ajh/hpx180, indexed in Pubmed: 29126128.

31. Colella TJF, Tahsinul A, Gatto H, et al. Antecedent rest may not be necessary for automated office blood pressure at lower treatment targets. J Clin Hypertens (Greenwich). 2018 [Epub ahead of print], doi: 10.1111/jch.13319, indexed in Pubmed: 29900674.

32. Armstrong D, Matangi M, Brouillard D, et al. Automated office blood pressure - being alone and not location is what matters most. Blood Press Monit. 2015; 20(4): 204-208, doi: $10.1097 /$ MBP.0000000000000133, indexed in Pubmed: 26154710.

33. Chambers LW, Kaczorowski J, O’Rielly S, et al. Comparison of blood pressure measurements using an automated blood pressure device in community pharmacies and family physicians' offices: a randomized controlled trial. CMAJ Open. 2013; 1(1): E37-E42, doi: 10.9778/cmajo.20130005, indexed in Pubmed: 25077100.

34. Paini A, Bertacchini F, Stassaldi D, et al. Unattended versus attended blood pressure measurement: Mean values and determinants of the difference. Int J Cardiol. 2019; 274: 305-310, doi: 10.1016/j. ijcard.2018.06.056, indexed in Pubmed: 29945805.

35. Myers M. Use of an automated blood pressurerecording device, the BpTRU, to reduce the "white coat effect" in routine practice. Am J Hypertens. 2003; 16(6): 494-497, doi: 10.1016/s08957061(03)00058-x, indexed in Pubmed: 12799100.

36. Beckett L, Godwin M. The BpTRU automatic blood pressure monitor compared to 24 hour ambulatory blood pressure monitoring in the assessment of blood pressure in patients with hypertension. BMC Cardiovasc Disord. 2005; 5(1): 18, doi: 10.1186/1471-2261-5-18, indexed in Pubmed: 15985180.

37. Myers MG, Godwin M, Dawes M, et al. Conventional versus automated measurement of blood pressure in the office (CAMBO) trial. Fam Pract. 2012; 29(4): 376-382, doi: 10.1093/fampra/ cmr113, indexed in Pubmed: 22117083.

38. Kollias A, Stambolliu E, Kyriakoulis KG, et al. Unattended versus attended automated office blood pressure: Systematic review and meta-analysis of studies using the same methodology for both methods. J Clin Hypertens (Greenwich). 2019; 21(2): 148-155, doi: 10.1111/jch.13462, indexed in Pubmed: 30585383.

39. Roerecke M, Kaczorowski J, Myers MG. Comparing Automated Office Blood Pressure Readings With Other Methods of Blood Pressure Measurement for Identifying Patients With Possible Hypertension: A Systematic Review and Meta-analysis. JAMA Intern Med. 2019; 179(3): 351-362, doi: 10.1001/ jamainternmed.2018.6551, indexed in Pubmed: 30715088. 
40. Schmieder RE. End organ damage in hypertension. Dtsch Arztebl Int. 2010; 107(49): 866-873, doi: 10.3238/arztebl.2010.0866, indexed in Pubmed: 21191547.

41. Campbell NRC, McKay DW, Conradson H, et al. Automated oscillometric blood pressure versus auscultatory blood pressure as a predictor of carotid intima-medial thickness in male firefighters. J Hum Hypertens. 2007; 21(7): 588-590, doi: 10.1038/ sj.jhh.1002190, indexed in Pubmed: 17377600.

42. Palomba C, Donadio S, Canciello G, et al. Unattended Automated Office Blood Pressure Measurement and Cardiac Target Organ Damage, A Pilot Study. High Blood Press Cardiovasc Prev. 2019; 26(5): 383-389, doi: 10.1007/s40292-019-00337-1, indexed in Pubmed: 31444783.

43. Salvetti M, Paini A, Aggiusti C, et al. Unattended versus attended blood pressure measurement: Mean values and determinants of the difference. Int J Cardiol. 2019; 274(3): 305-310, doi: 10.1016/j. ijcard.2018.06.056, indexed in Pubmed: 29945805.

44. Rabi D, McBrien K, Sapir-Pichhadze R, et al. Hypertension Canada's 2020 Comprehensive Guidelines for the Prevention,
Diagnosis, Risk Assessment, and Treatment of Hypertension in Adults and Children. Can J Cardiol. 2020; 36(5): 596-624, doi: 10.1016/j.cjca.2020.02.086, indexed in Pubmed: 32389335. 45. Tykarski A, Filipiak KJ, Januszewicz A, et al. Zasady postępowania w nadciśnieniu tętniczym — 2019 rok. Wytyczne Polskiego Towarzystwa Nadciśnienia Tętniczego. Nadciśnienie Tętnicze w Prakt. 2019; 5: 1-86.

46. Unger T, Borghi C, Charchar F, et al. 2020 International Society of Hypertension Global Hypertension Practice Guidelines. Hypertension. 2020; 75(6): 1334-1357, doi: 10.1161/HYPERTENSIONAHA.120.15026, indexed in Pubmed: 32370572.

47. Stergiou GS, Palatini P, Parati G, et al. European Society of Hypertension Council and the European Society of Hypertension Working Group on Blood Pressure Monitoring and Cardiovascular Variability. 2021 European Society of Hypertension practice guidelines for office and out-of-office blood pressure measurement. J Hypertens. 2021 [Epub ahead of print], doi: 10.1097/HJH.0000000000002843, indexed in Pubmed: 33710173. 